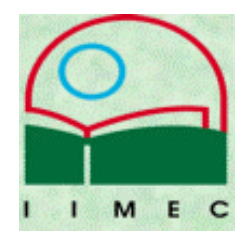

Universidad de Costa Rica

Facultad de Educación

Instituto de Investigación para el Mejoramiento

de la Educación Costarricense

ACTUALIDADES INVESTIGATIVAS EN EDUCACION

\title{
APORTES DE LA INVESTIGACIÓN CUALITATIVA Y SUS ALCANCES EN EL ÁMBITO EDUCATIVO.
}

\section{M.Ed. María Agustina Cedeño-Suárez}

En las siguientes líneas pretendo compartir algunas reflexiones sobre los alcances de la investigación como un proceso de búsqueda de conocimiento, interpretación y transformación de la realidad. En particular destaco los aportes que brinda la investigación cualitativa y etnográfica en el ámbito educativo, la cual abre un espacio para comprender la forma en que se construye en términos sociales y culturales la educación.

\section{La investigación: una forma de construir conocimiento}

En principio, la investigación debe ser reconocida como un proceso sistemático de aprendizaje orientado a conceptualizar la realidad, esto es, conocerla, analizarla y explicar sus manifestaciones. Toda investigación conlleva una intencionalidad, cual es, el mejoramiento y la transformación no sólo de las y los actores sociales que participan en ella, sino también de sus prácticas. Lógicamente esto exige una indagación constante por parte de las y los investigadores, con altas dosis de imaginación, intuición y deseo por acercarse y comprender la realidad estudiada.

Este proceso de construcción del conocimiento no es neutro. Los seres humanos logran transmutar la realidad desde su mera percepción sensorial y su visión del mundo, hasta categorizarla y analizarla mediante determinados enfoques o paradigmas, que permiten tanto la comunicación con otras personas, como la búsqueda de explicación de esos fenómenos reales.

No obstante, sería ilusorio pensar que para lograr lo anterior todas y todos los investigadores deban asumir el mismo paradigma de investigación: se debe elegir aquel que mejor convenga a sus intereses y a las exigencias del estudio que se llevará a cabo. El paradigma escogido va a condicionar los procedimientos de estudio que se fijen en la investigación, el tipo de problema elegido, las estrategias, técnicas e instrumentos, así como los criterios de validación y legitimación del conocimiento que se produce (Pérez Gómez, 
1990). El concepto que se tenga de realidad es un elemento que determina la elección de los procedimientos de investigación, y la utilización de ciertas estrategias metodológicas va a conducir a un tipo particular de conocimiento de la realidad estudiada.

Tomas Kuhn (1971) sostiene que el trabajo cotidiano de la ciencia se encuentra organizado en torno a un paradigma, el cual especifica la manera que tiene una comunidad científica de explicar y resolver problemas en un momento determinado. Cuando una comunidad científica se replantea su paradigma, se produce una crisis en la ciencia provocando una revolución científica; como resultado, dicha comunidad se reagrupa en torno a uno nuevo, aunque no se produzca un abandono radical del anterior ya que ambos tienden a coexistir.

Definido como una manera de hacer ciencia, el paradigma se visualiza de la siguiente manera:

"Un sistema de creencias, principios, valores, premisas que determinan la visión que una determinada comunidad científica tiene de la realidad, el tipo de preguntas que es legítimo estudiar, así como los métodos y técnicas válidos para la búsqueda de respuestas y soluciones. (T. Khun citado por Contreras, 1996)

Como conjunto de ideas, creencias y supuestos que permiten interpretar la realidad, así como los problemas específicos susceptibles de ser investigados, el paradigma proporciona un marco filosófico que posibilita estudiar de manera organizada la realidad; elabora reglas, definiciones, teorías que las comunidades científicas van articulando con base en la observación empírica. En consecuencia, es importante que las y los investigadores tengan claridad acerca de qué, cómo, para qué y por qué va a investigar determinada situación o fenómeno, ya que los propósitos, estrategias y procedimientos que elijan, deben ser congruentes con el paradigma en el cual se va a apoyar.

Normalmente se ha hecho una diferencia entre los paradigmas de investigación, la cual se ha sustentado en los atributos específicos que posee cada uno de ellos. Para Lincoln y Guba (1994) las diferencias entre los paradigmas se definen a partir de tres niveles relacionados entre sí: a. nivel ontológico, se refiere a la forma y la naturaleza de la realidad, a lo que es posible conocer; b. el nivel epistemológico, hace referencia a la naturaleza entre 
el sujeto que conoce y lo que puede ser conocido, por ende, a la posición que deben asumir las y los investigadores, como actores histórico sociales, respecto al objeto de conocimiento, y c. el nivel metodológico que se sustenta en la forma en que las y los investigadores proceden para hallar el conocimiento de la realidad que van a conocer, la cual tiene una identidad propia, histórica, dinámica, heterogénea y dialéctica.

Si bien a la luz del debate actual se tiende a buscar la complementariedad entre diversos paradigmas en el análisis de la realidad, es importante rescatar la premisa de que una investigación se ubica en uno u otro paradigma, por el diseño mismo de la investigación, por la lógica particular de razonamiento que establece el investigador desde el momento en que delimita el tema, plantea el problema, define los objetivos, hasta la elaboración del referencial teórico y metodológico que sustenta el estudio, para lograr el análisis e interpretación de los datos.

En el caso que nos ocupa, la investigación cualitativa nos acerca a un marco que posibilita compartir experiencias y desarrollar una relación dialógica con las y los actores sociales que participan en el estudio. Esto es, una mejor comprensión de sus experiencias vivenciales, su situación particular, su percepción de mundo y de las realidades que se obvian, porque se convirtieron en una acción rutinaria de acciones, organizadas en el aquí y ahora.

\section{Paradigma naturalista o cualitativo}

Tradicionalmente este paradigma se ha visto en oposición al cuantitativo (global, positivista, hipotético, deductivo, particularista), puesto que en las disciplinas del ámbito social existen diferentes problemáticas que no se pueden explicar ni comprender, en toda su extensión, desde ese enfoque. Principalmente estos nuevos planteamientos proceden de la antropología, la etnografía, y el interaccionismo simbólico.

Varias perspectivas y corrientes han contribuido a su desarrollo, cuyos supuestos coinciden en lo que se ha llamado paradigma hermenéutico, interpretativo-simbólico o fenomenológico. Los que impulsan estos supuestos fueron, en primera instancia la Escuela Alemana, con Dilthey, Husserl y Baden. También han contribuido al desarrollo de este paradigma autores como Mead, Schutz, Berger, Luckman y Blumer. (Pérez Serrano, 1994). 
Ahora bien, dado que el paradigma cualitativo ha sido objeto de múltiples estudios, en los cuales se destacan sus características y posibilidades, así como las bondades y ventajas que ofrece su utilización, entre otros aspectos, se debe insistir, como lo dice Valles (1997)

...en que no hay un único relato histórico que documente, de manera comprehensiva e indiscutible, la génesis y desarrollo de la perspectiva cualitativa en las ciencias sociales (ni en todas en conjunto, ni en ninguna de ellas por separado). (p.15)

Por su parte, Camacho-Zamora (1997) afirma:

La investigación cualitativa como producto de las actividades de acumulación de conocimiento en disciplinas como la antropología social y cultural o la sociología del interaccionismo simbólico -consideradas por otros enfoques y por la visión científica positivista prevaleciente como parientes pobres y marginales- se elaboró a lo largo del tiempo como un saber "esotérico." En ese sentido su aprendizaje, su manejo y su evaluación se constituyó en el campo de acción de una minoría de conocedores (p.1)

Las reflexiones de este tipo proporcionadas por diversos autores y puntos de vista, enriquecen la comprensión de esta tradición particular en la ciencia social, que depende de la comprensión y observación, por parte de las y los investigadores, de las personas en su propio contexto y de la interacción en su propio lenguaje y en sus propios términos. Bajo esa premisa, es posible señalar que este enfoque tiene un compromiso en el campo de actividades, y enfatiza en la relación y articulación de los diferentes elementos que conforman la totalidad.

Además, se considera como un proceso activo, sistemático y riguroso de indagación dirigida, en el cual se toman decisiones sobre lo que se investiga, en tanto se está en el campo de estudio. Algunos autores, entre ellos Erickson (citado por Pérez Serrano, 1994) indican que las y los investigadores entran en el campo con una orientación teórica consciente, que refleja un conocimiento sustantivo de la teoría de las ciencias sociales y de la teoría personal. Como producto de una interacción entre ambos, sugiere las preguntas y vías que orientarán la investigación. 
Quizá los aspectos más controvertidos en este paradigma y siempre sujetos a debate, han sido los criterios de veracidad de la información, es decir, la profundidad con la que es capaz de demostrar el rigor científico de sus explicaciones, construcciones y conocimientos (Angulo, 1990). En este sentido, la tradición cualitativa tiene elementos relevantes para el análisis y la comprensión de los fenómenos que se estudian.

El desarrollo y conceptos de categorías parten de los datos, por lo que no suelen probarse hipótesis o variables susceptibles de medición. Bajo esa premisa el compromiso con la objetividad no se fundamenta en someter el objeto de estudio a mediciones estadísticas, o la búsqueda de verdades finales o absolutas. Al respecto, Camacho Zamora (1997) apunta lo siguiente:

"Los supuestos que subyacen en la búsqueda de objetividad no son simples. Hay un mundo de realidad empírica ahí fuera de nosotros. La manera en que percibimos y entendemos ese mundo depende ampliamente de nosotros. Pero ese mundo externo no tolera todas las concepciones acerca de él mismo de la misma forma". (p:3).

La objetividad se evalúa en términos de la confiabilidad y la validez de las observaciones, aunque ambos elementos, herencia del paradigma positivista, no son de interés particular en la tradición cualitativa dada la forma en que se concibe, teoriza y conduce el trabajo de investigación. La confiabilidad (Camacho-Zamora, 1997) refiere al grado en que los hallazgos son independientes de las circunstancias accidentales de la investigación, y la validez al grado en que los hallazgos son interpretados de una manera adecuada para describir la realidad en estudio.

La confiabilidad se relaciona con las técnicas que utilizan las y los investigadores, y la validez se obtiene de la lógica de las interpretaciones, esto es, lo que las personas dicen. Ambos aspectos no son simétricos; es posible obtener confiabilidad sin que exista validez. Pero la validez asegura la confiabilidad, ya que cada observación, cada registro, conforma un esquema de lo que se ha captado. La observación y el trabajo de campo constituyen una prueba de validez. De ahí que, según Camacho-Zamora (1997), la tradición cualitativa:

"No busca alimentar con observaciones y datos de la realidad estudiada categorías pre-establecidas de acuerdo con la experiencia y mentalidad del observador. Por el contrario, busca poner en evidencia la manera en que los sujetos estudiados 
categorizan, jerarquizan y visualizan el mundo en que están inmersos. De esta forma, da cuenta de la experiencia del grupo en estudio y no de la sociedad en que se ubica el observador. " (p. 13).

Unido a lo anterior, otra forma alternativa de asegurar la exactitud de los resultados es mediante la triangulación, que permite combinar diversas fuentes de datos e instrumentos en el análisis de la información, evitando que las y los investigadores acepten de entrada la validez de sus primeras impresiones. La triangulación al menos permite tres importantes resultados: a. la convergencia, cuando por ejemplo, datos obtenidos por diferentes fuentes aportan evidencia confirmatoria de una conjetura que el investigador haya intentado comprobar; b. la inconsistencia, que, al contrario de la anterior, no confirma la conjetura; y c. la contradicción, que revela palpables contradicciones entre los datos, o las informaciones obtenidas. (Angulo, 1990). Es importante indicar que la triangulación se utiliza en la investigación cualitativa como una alternativa, pero nunca como estrategia o herramienta de validación.

A partir de estos planteamientos y con el aporte que ofrecen varios autores, entre ellos Filstead (1986), Pérez Serrano (1994) y Gurdián (1999), a continuación se exponen los principales supuestos en los que se basa el paradigma cualitativo.

- La teoría constituye una reflexión en y desde la praxis. La realidad está constituida por hechos observables y externos, por significados, símbolos e interpretaciones elaboradas por las personas en su interacción con los demás. Por lo tanto, se centra en la identificación de las reglas que subyacen y gobiernan los fenómenos sociales. Lo que se busca son patrones de intercambio, resultado de compartir significados e interpretaciones sobre la realidad.

- Intenta comprender, interpretar y explicar la realidad para transformarla. Subyace un fundamento humanista para entender la realidad; en su conocimiento inciden los valores del contexto social y cultural, por ello el conocimiento no es aséptico, ni neutro; se relaciona con los significados sociales, los símbolos y las interpretaciones de los seres humanos en el contexto de su interacción con los otros. No concibe al mundo como independiente del ser humano. 
- Describe e interpreta la situación en la que se desarrolla el acontecimiento. Se opta por la rigurosa descripción contextual de un hecho o una situación. Incorpora las percepciones, experiencias y creencias de los informantes, así como su propio lenguaje.

- Estudia a profundidad una situación concreta. No busca la explicación o la causalidad, sino la comprensión, y puede establecer inferencias plausibles entre los patrones de configuración en cada caso. Su objetivo es profundizar en el conocimiento de la vida social tal como ocurre.

- Profundiza en los diferentes aspectos que influyen en determinadas situaciones. La realidad es holística y polifacética, no es estática, esta se va creando. No existe una única realidad, sino múltiples realidades interrelacionadas; el ser humano es activo en la construcción y determinación de las realidades que encuentra; interesa conocerlas en su dimensión espacial y temporal, el aquí y el ahora en un contexto histórico social determinado.

- Los seres humanos son personas interactivas, comunicativas, que comparten significados. La relación sujeto/objeto es de interacción. Los significados mediante los cuales actúan las personas están mediatizados por sus formas de vida. Por eso, es necesario descubrir el conjunto de reglas sociales que dan sentido a un determinado tipo de actividad social.

- Las y los investigadores pueden reaccionar ante la realidad que estudian y los conocimientos que van adquiriendo, producto de las relaciones que establecen y los significados que comparten con las personas. Esto supone que deben ser capaces de conocerse a sí mismos y tratar de descubrir quiénes son como sujetos históricos frente a la realidad y a su objeto de conocimiento.

En cuanto a los procedimientos para tratar la información, este paradigma cualitativo acorde con el aporte de Filstead (1986) y Pérez Serrano (1994) presenta las siguientes características:

- Registra los datos de acuerdo con el lenguaje de los participantes. 
- Interesa que la teoría emerja de los propios datos; lo que incrementa la posibilidad de comprender y concebir una explicación del fenómeno en estudio.

- Se intenta descubrir que teorías, conceptos y categorías sugieren los propios datos, para dar sentido a la realidad social en la que se encuentran.

- Utiliza la aproximación a la vida cotidiana de las y los participantes para captar sus acciones.

- Para explicar el fenómeno en estudio emplea conceptos sensibles que captan el significado de lo que sucede. Los conceptos seleccionados se modifican con base en la recolección de datos, y en informaciones adicionales que respondan a nuevos interrogantes en el proceso de investigación

- Para obtener información recurre a observaciones, entrevistas en profundidad, entrevistas no estructuradas o semiestructuradas, estudio de casos, historias de vida.

El punto básico de partida del enfoque cualitativo está centrado en la comprensión de las personas dentro del marco de referencia de ellas mismas, en el contexto de su práctica concreta, de su cotidianidad. Esto sustenta el por qué la comprensión se valora desde una perspectiva holística, totalizadora, no fragmentada, que considera todos los puntos de vista como valiosos y dignos de estudiar; y donde las creencias, juicios de valor $y$ predisposiciones de las y los investigadores deben tratar de apartarse. De esta forma, ningún aspecto de la vida social resulta ser tan frívolo o trivial para no ser estudiado.

El interés por describir e interpretar la vida social y cultural de las y los participantes, los significados sociales desde la propia perspectiva de las y los actores y la insistencia en que sólo pueden ser examinados en el contexto de la interacción de las personas, es un aspecto que distingue a este paradigma del enfoque de las ciencias naturales.

En síntesis, es posible afirmar que el paradigma cualitativo explicita cuatro niveles interdependientes que lo fundamentan y condicionan (Gurdián, et.al, 1999): teleológico, ontológico-axiológico, epistemológico y metodológico. El nivel teleológico responde a la finalidad del problema de investigación, es decir, al para qué; en el nivel ontológicoaxiológico, relación con la naturaleza de la realidad, se asume la existencia de múltiples realidades construidas desde diferentes perspectivas, realidad en constante transformación que se puede estudiar holísticamente; donde se trata de comprender, descubrir y comunicar 
los significados de los actores sociales. En el aspecto epistemológico, centrado en la relación entre quien conoce y lo que puede ser conocido, las y los investigadores asumen una posición frente al objeto de conocimiento, en la definición del problema, en su delimitación. También asumen una posición frente a la interacción que establece con las y los informantes al tratar de transformar la realidad y construirla de manera colectiva. El nivel metodológico, que se sustenta en las variadas formas que se buscan para encontrar el conocimiento de la realidad, posibilita la complementariedad de técnicas para estudiar a profundidad el problema elegido y realimentar la información obtenida para darle su pleno significado.

Los aportes que ha brindado el paradigma cualitativo para comprender diversos aspectos de la vida humana, se han dejado sentir en el ámbito educativo. Particularmente, la investigación etnográfica, ha abierto un espacio para la reconstrucción cualitativa de procesos y relaciones educativas, con la intención de comprender la forma en que se construye en términos sociales y culturales la educación, temática a tratar seguidamente.

\section{La investigación etnográfica}

La etnografía se ha caracterizado por describir y analizar en detalle situaciones, eventos, personas, interacciones y comportamientos observables, así como por documentar lo que los participantes dicen, sus experiencias, actitudes, creencias, pensamientos y reflexiones tal y como son expresadas por ellos mismos en su propio contexto.

El término proviene de la antropología y deriva de las palabras griegas ethnos (pueblo, grupo humano) y graphein (describir) por lo que refiere a la descripción o reconstrucción de escenarios y grupos culturales, esto es, su estilo de vida, costumbres, valores, comportamientos y creencias.

Bajo una perspectiva etnográfica se pretende crear una imagen real y fiel del grupo que se estudia, la búsqueda de la intencionalidad y del significado de las acciones o de las situaciones que emergen en contextos específicos. El referente empírico de los estudios etnográficos queda circunscrito en el horizonte de la concepción de mundo y de las representaciones ideológicas que tienen tanto las y los investigadores como las y los 
participantes, y de sus interacciones cotidianas y personales, para descubrir los significados que estos últimos dan a su actuar, a su vida misma.

Específicamente, se trata de aprender el mundo empírico de manera holística y natural, es decir, conocerlo en su totalidad, buscar las relaciones y articulaciones entre las partes y la totalidad, para comprender y analizar a las y los participantes en el contexto en que se insertan, reconstruir los aspectos no documentados, rescatar la cotidianidad, lo inconsciente, lo oculto de la realidad estudiada.

En ese sentido, el trabajo etnográfico se sustenta en los siguientes principios:

- Requiere de la participación y convivencia de las y los investigadores en la vida cotidiana de las y los participantes, mediante un trabajo de campo intensivo y sistemático, la observación y entrevistas a profundidad como técnicas primordiales de recolección de datos.

- Depende de la observación, por parte de las y los investigadores, de las personas en su propio contexto y de la interacción en su propio lenguaje y en sus propios términos.

- Parte de una interacción cara a cara con las y los participantes para explicar cómo el ser humano percibe, siente, vive, cambia, actúa, y construye y reconstruye su propia identidad.

- Pretende revelar la riqueza y diversidad de la experiencia humana, más que determinar las leyes que la gobiernan.

- Supone que la realidad social es construida por cada persona en interacción con todas las demás, y en relación con los significados y los símbolos que estos le otorgan.

- Parte de una concepción de totalidad, que visualiza la realidad como un proceso articulado y dialéctico, en donde un elemento puede ser conocido en su articulación con el todo.

- Enfatiza en las relaciones y articulaciones de los diferentes elementos que conforman la totalidad, y no las mediciones de las partes que se suponen conocidas y regulares.

- Complementa los aspectos émico y ético. Desde el punto de vista émico se buscan los símbolos, los pensamientos y la conducta de las y los participantes, con una actitud libre de conceptos apriorísticos, ya que todo lo dicen y hacen tiene sentido y es importante. Desde la perspectiva ética, las y los investigadores trascienden la conciencia de las y los participantes, para llegar a los aspectos no intencionales del ser humano y construir pautas culturales. 
- Como estrategia de investigación, se elabora en función de cada investigación particular y de los datos que se necesitan para su análisis. No es posible su aplicación de modo mecánico en otras realidades.

- Permite delinear y contextualizar las prácticas culturales locales y la forma en que los seres humanos organizan su cultura.

- Utiliza un proceso cíclico de investigación. En dicho proceso las y los investigadores tienen sucesivas oportunidades para precisar, definir y ordenar su estudio con el fin de buscar la coherencia entre las interpretaciones de los participantes y la realidad. Por lo tanto, no se requiere la formulación de hipótesis prediseñadas o esquemas rígidos antes de iniciar el trabajo de campo. La teoría emerge de la realidad.

- Es flexible, con capacidad de adaptación a los contextos en que se utiliza. Las y los investigadores pueden emplear diferentes técnicas de recolección de la información, entre ellas: observación participativa, observación no participativa, entrevistas abiertas a profundidad, historias de vida y análisis de diversos documentos.

Específicamente, en el contexto de la educación la etnografía, denominada etnografía educativa, pretende la descripción e interpretación detallada, profunda y analítica de las actividades, creencias compartidas, prácticas y procesos que intervienen en la constitución de lo escolar y en su cotidianidad, desde la perspectiva de las y los participantes y del contexto social y cultural en que estos se desenvuelven.

\section{Investigación etnográfica en educación}

La etnografía educativa propicia un tipo de acercamiento que permite aprehender las particularidades de las relaciones sociales que se establecen en el contexto escolar, ya que parte de que la realidad se construye socialmente; no es por lo tanto, independiente de los seres humanos. (Bertely, 1997; Getz y Le Compte 1988). Sin embargo, no es sino hasta finales de los años setenta que la investigación etnográfica/cualitativa empieza a 'legitimarse', es decir, goza de aceptación en diversos campos disciplinarios, incluyendo el educativo. Anteriormente (Bertely, 1997; Bertely y Corenstein, 1994), no se brindaba atención a la dinámica interna de las instituciones educativas, la práctica educativa cotidiana o las interacciones en el aula; el desempeño escolar fue abordado, principalmente, desde un punto de vista cuantitativo y experimental, donde predominan las pruebas estandarizadas y las explicaciones parciales que no dan cuenta del contexto social y cultural en que se desenvuelven los estudiantes. 
En términos generales, la década de los ochenta marca un cambio sustancial en la investigación etnográfica en educación. La problemática educativa empieza a ser tratada desde referentes conceptuales críticos (Rockwell,1991; LeCompte,1995) que pretenden señalar la influencia que ejercen los factores y las condiciones sociales, económicas y culturales en las desigualdades entre las personas y los grupos, particularmente aspectos tales como clase social, etnia, procedencia geográfica y género. Esta nueva orientación permite, por ejemplo, abandonar la idea de que el creciente fracaso escolar, la deserción y el analfabetismo son producto de los estudiantes y sus familias, y no de la estructura económica y social.

La crítica a la escuela se respalda fundamentalmente en las llamadas teorías de la reproducción que vinculan los procesos escolares con la reproducción del sistema social. Bajo esta concepción, se concibe la escuela como un aparato ideológico de dominación que no enfrenta conflictos ni resistencias ante lo establecido. Su discurso se dirige a otorgarle a la institución escolar un carácter neutral, que tiene la función de reproducir jerarquías, normas, valores y conocimientos, con el fin de socializar a las personas y prepararlas para que se incorporen al sistema productivo. Por lo tanto, la escuela se convierte en un sitio de instrucción que legitima los intereses del orden social imperante, y desarrolla mediante un currículo oculto, procesos de socialización autoritaria amparada en una ideología igualitaria y de control social.

En suma, se deja entrever que la cultura escolar está mediatizada por intereses y conflictos sociales y culturales que se manifiestan en las acciones y en las prácticas cotidianas de los actores del proceso educativo. Parafraseando a Giroux, las escuelas se convierten en sitios de "dominación y resistencia", de subordinación y crítica, contestación y lucha entre diferentes grupos; sitios en los que se construyen y reconstruyen contenidos culturales y relaciones sociales. En esta línea sobresalen los trabajos autores tales como: Althusser; Bordieu y Passeron, Baudelot y Establet; Bowles y Gintis, y Apple.

De ahí que las concepciones sobre el carácter reproductor de la escuela, se dejan sentir en las investigaciones que se desarrollan en América Latina en el campo educativo, y se vuelve la mirada hacia los sectores populares y su participación en la educación. 
Resulta interesante señalar que, paralelamente, bajo la perspectiva etnográfica crítica, se aboga para que los etnógrafos no sólo incidan en la transformación de las condiciones de vida de los grupos que estudian y logren concientizarlos de su situación, sino también para que documenten sus propias vivencias en el trabajo de campo, sus relaciones y los cambios que experimentan en su contacto cotidiano con el grupo. Con ello se procura darle mayor sentido y significado a la vida y a las concepciones de mundo de los miembros del grupo, y de las y los investigadores, haciendo visible su trabajo, implicado en lo que podríamos llamar 'una acción emancipatoria.'

En tanto, las reflexiones que se elaboran sobre el contexto educativo tienden a recuperar de manera paulatina las dimensiones sociales y culturales del trabajo escolar. Se incursiona, entre otros aspectos, en el proceso de enseñanza aprendizaje; las formas que adopta la educación en diversas culturas y grupos sociales; la vida cotidiana del aula para comprender las interacciones y el significado que docentes y alumnos conceden a su realidad y al contexto en que se ubican. La metodología básica utilizada para recopilar los datos continúa siendo la observación participativa, la observación no participativa, entrevistas abiertas a profundidad, historias de vida y análisis de diversos documentos.

Asimismo, se analiza el papel de la escuela en torno a la construcción de la identidad genérica, que se traduce en la experiencia de las personas en torno a su ser y existir. Los seres humanos construyen una forma de ser y de percibirse a sí mismos y en relación con otras personas; en dicha construcción son fundamentales el intercambio y las asignaciones sociales y culturales con las cuales la historia los ha constituido en mujeres y hombres, una constitución que va más allá de la biología, pues incide en cómo se perciben, se sienten y se conciben como seres humanos. Así pues, la identidad genérica no es una determinación biológica o psicológica, la construimos a partir de nuestras continuas interacciones con el medio que nos rodea, es por lo tanto, una construcción socio-cultural, pero no exclusivamente ideológica en la organización social que define posiciones, funciones y papeles a las y los seres humanos, sino también se apropia en la existencia y en la vivencia personal, por lo que no hay una única forma de constituir la identidad genérica.

En el escenario educativo, docentes y estudiantes en sus relaciones, creencias y actitudes muestran una identidad genérica, mediante las interacciones que se construyen en la cotidianidad de la escuela y del aula. En ese sentido, son las experiencias y la variedad de 
ellas las que nos brindan la oportunidad de conformar, paulatinamente, nuestra identidad y reestructurarla al confrontar la visión de mundo que poseemos con lo que nos proyecta la realidad. Esta línea parece ser la que orienta muchos de los trabajos que, bajo una perspectiva etnográfica, analizan la categoría de género.

\section{Escenario educativo y estudios de género}

Los estudios sobre género en el ámbito educativo han logrado ocupar un sitio destacado. Existe literatura que desde la perspectiva antropológica o sociológica intenta encontrar explicaciones a las desigualdades existentes entre hombres y mujeres, basadas en las nociones de clase, raza y género. (Mills, 1997).

El género se ha visto como una categoría que involucra distintos enfoques: psicológicos, biológicos, culturales, sociales e históricos. Funciona bajo el eje de la diferencia biológica y psicológica, y en el ámbito socio-cultural, político y económico se representa en términos de desigualdad. La antropología propone el estudio del género como construcción cultural, e incorpora, como lo plantea Beltrán (1997) "determinaciones sociales y culturales, con la valoración propia de la disciplina, de los contextos locales, concretos y particulares, en que las relaciones entre los géneros tienen existencia". (p.2)

La idea general mediante la cual se distingue "sexo" de "género" consiste en que el primer término refiere al hecho biológico de que la especie humana es una de las que se reproduce a través de la diferenciación sexual, mientras que el segundo término, guarda relación con los significados que cada sociedad atribuye a esa diferenciación. Es decir, cada cultura elabora y desarrolla esta categoría de diversos modos, y asigna papeles, posiciones y lugares determinados a los hombres y a las mujeres, generando un sistema desigual. Además, está ligado a otros aspectos determinantes de la vida de las personas como son, entre otros: historia familiar, oportunidades educativas, nivel socioeconómico, forma de ser de los hombres y de las mujeres.

Una de las ideas más importantes acerca de la concepción de género, es que los modos de pensar, sentir y comportarse de hombres y mujeres, más que tener una base natural e invariable, se apoyan en construcciones sociales que aluden a características culturales y psicológicas asignadas de manera diferenciada. Por medio de tal asignación, mediante los recursos de la socialización temprana, unos y otras incorporan ciertas pautas de 
configuración psíquica y social que hacen posible el establecimiento de la identidad femenina y masculina.

Desde ese punto de vista, el género se define como red de creencias, rasgos de personalidad, actitudes, sentimientos, valores, conductas y actividades que diferencian a hombres y mujeres. Tal diferenciación es producto de un largo proceso histórico de construcción social, que no sólo genera diferencias entre los géneros femenino y masculino sino que, a la vez, esas diferencias implican desigualdades y jerarquías basadas en relaciones de poder entre ambos. (Burin, 1996).

Tal como lo señala Gurdián (2001) las relaciones de poder se refieren a todas las relaciones existentes entre seres humanos en las que unas personas tratan de orientar, conducir e influir en las y los otros. Si bien estas relaciones adoptan distintas formas y se producen en niveles diferentes, no se fijan de antemano, por lo tanto son susceptibles de modificar e incluso transformar.

Dentro de una cultura, mujeres y hombres cumplen diferentes papeles y posiciones sociales en determinadas etapas de sus vidas como personas y ámbitos donde se desenvuelven, por ejemplo: niñas, madres, esposas, hermanas, hijas, niños, padres, esposos, hermanos, hijos, profesionales. Esto podría verse reflejado en las actitudes e interacciones que los estudiantes manifiestan en el contexto escolar, las cuales podrían estar representando, implícitamente, una visión de mundo sobre género propia de su cultura.

Sin embargo, la problemática sobre el género puede retomar otros puntos de vista, que van más allá del cumplimiento de papeles dentro de la sociedad. En este caso, es necesario trazar un puente que acerque el problema de género a la forma de aprender, y si esta forma logra distinguir a varones y a mujeres cuando construyen su conocimiento y asumen una posición frente al mismo.

Esta situación viene a ampliar el marco de referencia sobre género para ubicarlo en el escenario escolar. La escuela como agencia en donde se institucionaliza el proceso educativo, reproduce las relaciones de género. Esta reproducción se refleja en el currículo escolar - manifiesto u oculto -, en las prácticas educativas cotidianas y, en los procesos de interacción y mediación pedagógica que se generan en el aula. La idea de currículo oculto, ampliamente abordada por diferentes autores, está más allá de los conocimientos y las enseñanzas intencionales declaradas en las escuela y el aula, e incluye todo lo que se refiere a la inculcación de valores, actitudes y juicios acordes con el sistema sociocultural 
vigente. La noción de 'oculto' refleja el carácter implícito en el discurso formal, pero que se proyecta casi siempre de manera inconsciente en el ámbito de la cultura escolar, y en la acción pedagógica cotidiana a través de múltiples mensajes y formas de comunicación e interacción.

El aula se constituye en un espacio de intercambio socio-cultural donde convergen no sólo lenguaje, visión de mundo, actitudes, valores, sentimientos, emociones y, creencias de docentes y alumnos, sino también un conjunto de situaciones que tienen un significado particular, están enmarcadas en un tiempo y espacio específico y, se ejecutan con una finalidad determinada.

En estas interacciones e intercambios simbólicos, se revela el proceso de construcción de género y la manera en que se actúa. En este sentido, el discurso del aula se constituye en una praxis socio-cultural legitimadora, que debe comprenderse para transformarse. De esa forma, la escuela tiene que convertirse en un espacio deslegitimador de dicha visión; ser un espacio propositivo de cambio socio-cultural en relación con las creencias, valores, actitudes y concepciones que como mujeres y hombres tenemos.

En la actualidad la investigación etnográfica brinda valiosos aportes para explicar el papel que desempeña el género en la configuración de diversas experiencias en el contexto escolar y la cotidianidad del aula, a fin de lograr una educación más igualitaria entre géneros, que contribuya a eliminar actitudes y comportamientos sexistas y discriminatorios que se transmiten culturalmente.

Entre otras temáticas, sobresalen las siguientes:

- Las diferencias que se establecen entre hombres y mujeres para acceder al conocimiento. El interés se centra en determinar, por ejemplo, las diferencias entre hombres y mujeres en torno a la resolución de problemas de matemática, el conocimiento que tienen acerca de los problemas de género, la lectura de textos de educación sexual, la lectura, escritura y uso del lenguaje en idiomas extranjeros, el manejo de equipos de cómputo, la incidencia de la matemática en la ansiedad de los estudiantes, las construcciones que sobre género, raza, clase y etnia, hacen los estudiantes.

- Diferencias de género en cuanto a igualdad de oportunidades de educación. Los autores que analizan esta temática (Ramos et. al., 1996) plantean que la igualdad de género es 
más que poner a hombres y mujeres en iguales condiciones, para eliminar las barreras y los estereotipos que limitan las oportunidades y escogencias en ambos sexos.

- Diferencias de género en término de actitudes. Los estudios se interesan en conocer las actitudes hacia determinadas áreas del conocimiento, sobre todo las Ilamadas 'ciencias duras', entre ellas la matemática y las ciencias.

- Papel de las y los docentes en el aula. Hay un interés explícito en algunos estudios por demostrar que el papel que asuma el docente en la cotidianidad del aula, va a permitir que se promueva o no la igualdad entre géneros. De ahí la necesidad de buscar determinadas estrategias didácticas para lograr un cambio en las actitudes y comportamiento de los estudiantes, es decir, concientizarlos de que las condiciones de discriminación y subordinación entre géneros son una creación 'arbitraria', que responde a una sociedad patriarcal.

No cabe duda la trascendencia que tienen estos estudios en la construcción de una cultura equitativa de género en el ámbito educativo en general y particularmente, en la práctica cotidiana docente, espacio en el que se requiere establecer procesos de reflexión y diálogo, para que las y los actores educativos cimienten, sobre bases más sólidas, una nueva forma de interacción entre géneros. 


\section{Referencias}

Angulo Rasco, Félix. 1990. Una propuesta de clasificación de las técnicas de investigación interpretativa. En Hacia un enfoque interpretativo de la enseñanza. Etnografía y Curriculum. España: Universidad de Granada.

Bertely, M. y M. Corenstein. 1994. Panorama de la investigación etnográfica en México: una mirada a la problemática educativa. En: Rueda M; G. Delgado, Z. Jacobo (Coord.) La etnografía en educación. Panorama práctica y problemas. Coord. México: CISE-UNAM. p. 173-209

Bisquerra, Rafael. 1989. Métodos de investigación educativa. Guía práctica. Barcelona: Ediciones CEAC

Burin, M. 1996. Género y psicoanálisis: subjetividades femeninas vulnerables. En Burin, M. \& Dio Bleichmar, E. (Comp.) Género y psicoanálisis, subjetividad. Buenos Aires, Argentina: Paidós. p. 61-100

Camacho-Zamora, José Antonio. 1997. Investigación cualitativa y sus aplicaciones. Mimeo. Costa Rica.

Colás B., Ma. Pilar, Buendía E. Leonor. 1994. Investigación educativa. Sevilla, España: Ediciones Alfar.

Contreras Montes de Oca, lleana. 1996. La investigación en el aula en el marco de la investigación cualitativa en educación: una reflexión acerca de sus retos y posibilidades. Revista de Educación, Vol. 20 (1), 109-125.

Cook, T. D. \& Reichard S. 1986. Métodos cualitativos y cuantitativos en investigación evaluativa. España: Ediciones Morata.

Filstead, William. 1986. Métodos cualitativos, una experiencia necesaria en la investigación evaluativa. Métodos cualitativos y cuantitativos en investigación evaluativa. España: Ediciones Morata.

Goetz, J.P. y LeCompte, M. 1988. Etnografía y diseño cualitativo en investigación educativa. Madrid: Ediciones Morata.

Gurdián, Alicia, et al. 1999. El paradigma cualitativo. Universidad de Costa Rica: Instituto de Investigaciones para el Mejoramiento de la Educación (IIMEC).

. 2001. Tocando la puerta y dejando el recado... ¿Cómo incluir la equidad de género en un proyecto curricular en Derecho?. Universidad de Costa Rica: Instituto de Investigaciones para el Mejoramiento de la Educación (IIMEC). Mimeo. En prensa.

Jiménez Fallas, Kembly. 1999a. Algunas reflexiones teóricas para la investigación en el aula. Universidad de Costa Rica. Departamento de Docencia Universitaria. Mimeo 
Kuhn, Tomas. 1971. La estructura de las revoluciones científicas. México: Fondo de Cultura Económica.

LeCompte, Margaret. 1995. Un mensaje personal desde los Estados Unidos: la etnografía educativa de la periferia hasta la corriente central; de la estructura al caos. Mimeo. Estados Unidos.

Lincoln, Y; E. Guba. 1994. Competing paradigms in qualitative research. En: Norman Denzing e Ivonna Lincoln (Editores). Handbook of qualitative research. California: Sage Publications.

Mills, A. 1997. Gender, Bureaucracy, and the business curriculum. Journal of Managent Education, 21, (3) August, 325-342.

Pérez Serrano, Gloria. 1994. Investigación cualitativa. Retos e interrogantes. Madrid, España: Editorial La Muralla S. A.

Pérez Gómez, Angel. 1990. Comprender y transformar la enseñanza. Madrid, España: Ediciones Morata.

Ramos, Ismael; Lambating, Julia. 1996. Risk taking: Gender differences and educational opportunity. School Science \& Mathematics. Vol. 96 (2), feb. 94-98.

Rockwell, E. 1991. Enfoque crítico de la escuela en América Latina. Perspectivas. pp. 111, UNESCO.

Taylor, S. J. \& R. Bodgan. 1992. Introducción a los métodos cualitativos de investigación. La búsqueda de significados. Barcelona: Ediciones Paidós

Valles, Miguel S. 1997. Técnicas cualitativas de investigación social. Reflexión metodológica y práctica profesional. Madrid. España: Editorial Síntesis S.A.

\section{Referencias telemáticas}

Beltrán, J. 1997. Sobre género y educación. Recuperado en febrero del 2001 de http://www.e-h.uv.mx/pubjeny.htm.

Bertely, María. 1997. Retos metodológicos en etnografía de la educación. Recuperado en marzo del 2001 de http://www.e-h.uv.mx/PUBLMARI.htm. 


\section{Dimensiones de la práctica docente}

Acorde con el planteamiento de Fierro y otras (1999) entre las dimensiones que conforman la práctica del docente se encuentran las siguientes: personal, interpersonal, social, institucional, didáctica y valoral. Los elementos que conforman cada dimensión se exponen a continuación.

\section{Dimensiones de la práctica docente*}
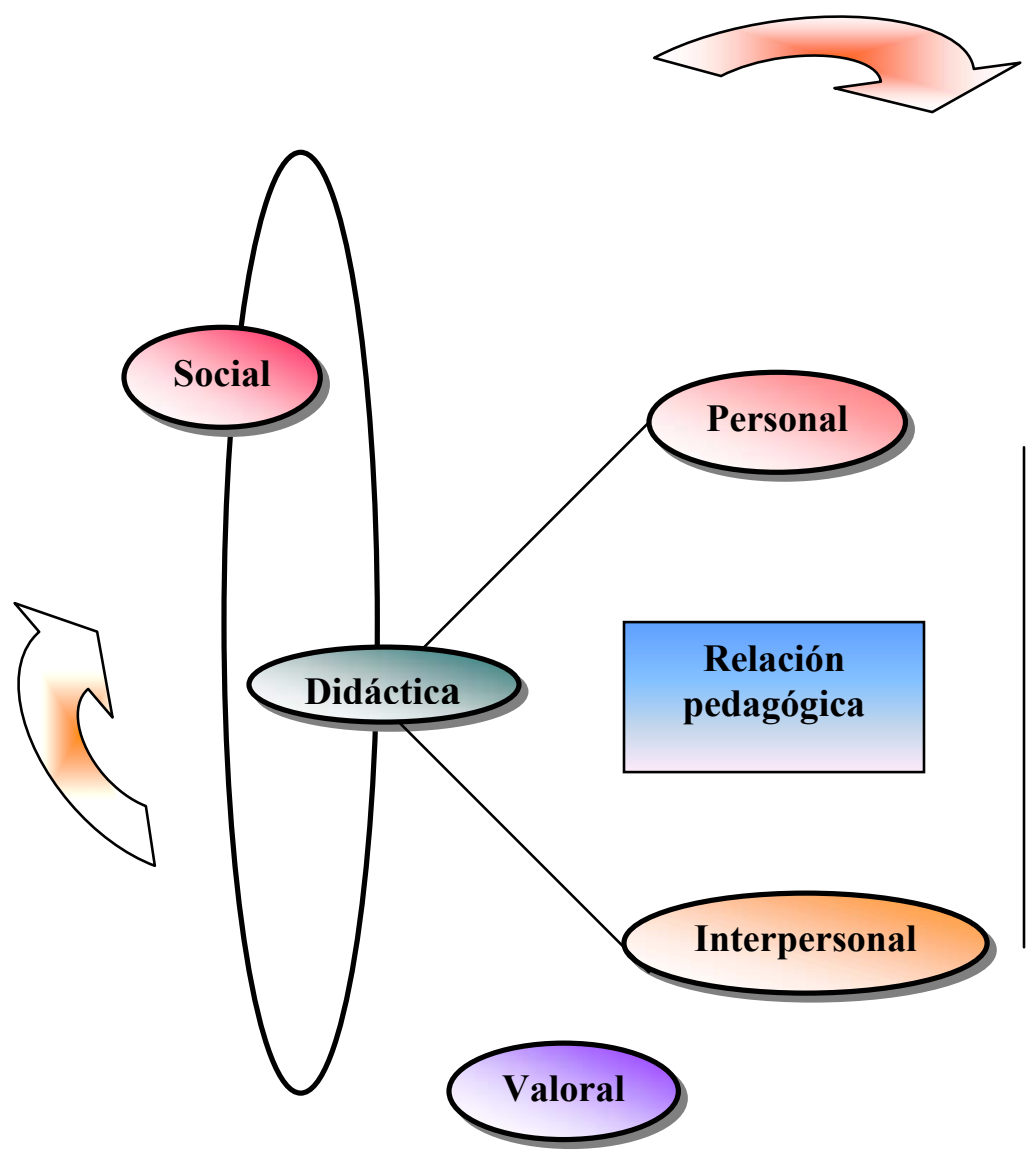

Institucional

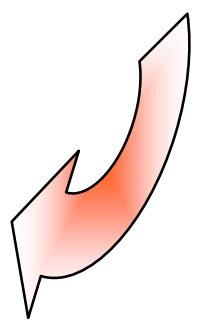

${ }^{*}$ Con base en Fierro y otras (1999) 


\section{- Dimensión personal}

La figura del docente es uno de los ejes que vertebra la práctica educativa. En esta dimensión sobresale la necesidad de que se reconozca a sí mismo como sujeto histórico, con características particulares y con capacidad de analizar su presente. Al analizar esta dimensión, el docente debe recuperar la forma en que se unen su historia personal y su trayectoria profesional; su vida cotidiana y su trabajo docente, es decir, quien es fuera del salón de clases, lo que representa su trabajo y la manera en que su vida privada se hace presente en el aula. Además, requiere tener presente las experiencias profesionales que han sido significativas en su vida, sus sentimientos de éxito o fracaso profesional, lo que se propone lograr, así como la visión prospectiva de su profesión.

\section{- Dimensión institucional}

La práctica docente se desarrolla en el seno de una organización. En ese sentido el quehacer docente es una labor construida colectivamente y regulada en un contexto escolar particular, en el cual el docente entra en contacto con los saberes, tradiciones, costumbres y reglas de la cultura institucional. Por lo tanto, esta dimensión apunta a reconocer que las decisiones y las prácticas de cada docente están tamizadas por la experiencia institucional, en tanto hay un marco normativo y organizacional que influye en los criterios del trabajo docente.

\section{- Dimensión interpersonal}

La función del docente se asienta en las relaciones que establece con las personas que participan en el proceso educativo. Estas relaciones son complejas, pues se construyen sobre la base de las diferencias individuales, en un marco institucional particular. La manera en que se entretejen las relaciones interpersonales hace alusión al 'clima institucional' o ambiente de trabajo que prevalece en el centro educativo. En un clima institucional óptimo prevalece el respeto, la colaboración y la participación para la toma de decisiones. En contraste, un clima hostil e indiferente, empobrece (Fierro y otras, 1999) las posibilidades de actuación de los docentes e influye en el desempeño de los estudiantes.

En ese sentido, esta dimensión insta a los docentes a considerar la satisfacción que sienten por el trabajo, las estructuras y estilos de participación, el tipo de comunicación formal e informal que se establece, la motivación de los distintos actores educativos, así 
como los problemas que se presentan y cómo se resuelven para lograr los objetivos propuestos.

\section{- Dimensión social}

La labor docente es un quehacer que se inserta en un contexto histórico social y cultural determinado, el cual demanda una serie de condiciones a las instituciones educativas. En particular, como lo anota Fierro y otras (1999) esta dimensión intenta recuperar un conjunto de relaciones que se refieren a la forma en que cada docente percibe y expresa su tarea como agente educativo, cuyos destinatarios presentan condiciones socioeconómicas y culturales diferentes, colocándolos en una situación desigual frente a la tarea escolar. De ahí que en esta dimensión el análisis se centre no sólo en las especificidades que adquiere la práctica docente en un entorno particular, sino también en el análisis de las expectativas y las presiones que ejerce el sistema y los destinatarios en el docente.

El punto inicial para comprender esta dimensión es partir de que las instituciones educativas no son lugares neutros, no se limitan simplemente a transmitir de manera objetiva un conjunto común de valores y conocimientos. Por el contrario, representan formas de conocimiento, relaciones sociales y valores que implican selecciones y exclusiones particulares a partir de cultura general, y por ende, legitiman intereses políticos, económicos y socio-culturales particulares. Es por ello, que los docentes requieren crear las condiciones estructurales para investigar su propio desarrollo curricular.

\section{- Dimensión didáctica}

Esta dimensión hace referencia al papel del docente como agente educativo que orienta, facilita, dirige y guía la enseñanza de los alumnos, para que construyan su propio conocimiento. En este ámbito, la tarea del docente consiste en facilitar el acceso al conocimiento, y para ello debe saber que son capaces de aprender los alumnos, sus hábitos de trabajo, actitudes y valores frente a la realidad en que se desenvuelven. De las decisiones y prácticas de cada docente dependerá que el proceso de enseñanza se reduzca a la simple transmisión y repetición de la información, o constituya una experiencia enriquecedora y constructiva. La forma en que organiza el trabajo con los alumnos, conduce las situaciones de aula, los métodos que utiliza, las normas que rigen el trabajo de aula, el tipo de evaluación que emplea, así como la manera en que enfrenta los problemas académicos, son algunos de los aspectos que cobran relevancia en esta dimensión. 


\section{- Dimensión valoral}

En la práctica docente subyace la dimensión axiológica, es decir, un conjunto de valores. La institución educativa es portadora de valores, creencias, visiones de mundo, normas de comportamiento y de actuación semejantes en función de las expectativas e intereses individuales y grupales. Asimismo, cada docente tiene una forma particular de ver y entender el mundo, las relaciones humanas, el conocimiento y las experiencias de enseñanza. De ahí que tanto el proceso educativo como la práctica pedagógica no sean neutrales; ejercen determinada influencia en la formación de los alumnos, en su modo de actuar y de pensar.

Esta dimensión de la práctica docente posibilita analizar los valores de los docentes, que dan un significado especial a su vida profesional. Por ello la necesidad de plantearse en qué medida la propia práctica refleja los valores asumidos, enriqueciéndolos o relegándolos para caer en la acción rutinaria. A partir de este nivel, es posible analizar la vida cotidiana de la escuela, para descubrir los valores que subyacen en ella y como los asumen sus miembros.

\section{- Relación pedagógica}

Es importante mencionar que el nivel denominado 'relación pedagógica' expresa la forma en la que el docente integra y armoniza los elementos de cada dimensión. La actitud que asuma en este proceso, le permitirá o no reconocer contradicciones, equivocaciones o aciertos, y sobre todo, reflexionar de manera crítica acerca de sus propias acciones y transformar su práctica cotidiana.

Con base en los planteamientos realizados en este trabajo, se pretende aportar algunos lineamientos que orienten al docente en la reflexión de su práctica concreta, independientemente del paradigma que elija para realizar la investigación. 